\title{
聚合物材料表面原子氧防护技术的研究进展
}

\author{
李昊耕 ${ }^{1,2}$, 谷红宇 ${ }^{1}$, 章俞之 ${ }^{1,2}$, 宋力昕 ${ }^{1,2}$, 吴岭南 ${ }^{1}$, 齐振一 ${ }^{1}$, 张 涛 $^{1}$
}

(1. 中国科学院 上海硅酸盐研究所, 中国科学院特种无机涂层重点实验室, 上海 200050; 2. 中国科学院大学 材 料与光电研究中心, 北京 100049)

摘 要: 聚合物材料具有质量轻、强度高等优点, 常被用作航天器表面的复合结构基材。原子氧是低地球轨道空间 中成分含量最高的粒子之一, 对暴露在航天器表面的聚合物材料易形成大通量、高能量轰击, 造成其表面氧化侵蚀 和质量损失, 使聚合物材料的性能发生不同程度的衰退, 也是导致航天器件可靠性降低、工作寿命缩短的主要环境 因素。本文对当前国内外通用的几种聚合物材料表面原子氧防护技术进行了整理归纳, 其中表面化学改性方法结合 了体材改性和常用防护涂层的优点, 得到的有机/无机复合改性防护层具有较好的综合防护性能。文中分析了近年 来由计算模拟法开展原子氧与表面防护材料相关作用机理的研究, 指出采用计算模拟结合试验的研究方法, 有可 能从本质上揭示复合改性层与原子氧的作用机理, 从而促进原子氧防护材料与防护技术的研究发展。

关 键 词: 原子氧; 聚合物; 空间防护; 表面改性; 聚酰亚胺; 计算模拟; 综述

中图分类号: V54 文献标识码: A

\section{Surface Protection of Polymer Materials from Atomic Oxygen: a Review}

\author{
LI Hao-Geng ${ }^{1,2}$, GU Hong-Yu ${ }^{1}$, ZHANG Yu-Zhi ${ }^{1,2}$, SONG Li-Xin ${ }^{1,2}$, \\ WU Ling-Nan ${ }^{1}$, QI Zhen-Yi ${ }^{1}$, ZHANG Tao $^{1}$
}

(1. Key Laboratory of Inorganic Coatings Materials CAS, Shanghai Institute of Ceramics, Chinese Academy of Sciences, Shanghai 200050, China; 2. Center of Materials Science and Optoelectronics Engineering, University of Chinese Academy of Sciences, Beijing 100049, China)

\begin{abstract}
Polymers, as substrate of composite material on the surface of spacecraft, have such advantages as light mass and high strength. Atomic oxygen (AO) is one of the highest content particles of low earth orbit, and high-energy high-flux AO bombardment causes the polymers' surface erosion and mass loss at different degree, resulting in polymers degradation. Thus, AO is one of major threats in space environment that reduces reliability of space devices and shortens their working life span. This review summarized current global protection technologies from $\mathrm{AO}$ in recent years. Among them, surface chemical modification method with advantages of body-modification and protection coating, provided organic/inorganic composite with modified layer through comprehensive protection performance. This review discussed the method to explore mechanism of the AO protection reaction by computational simulation. Computational simulation combined with experiments may reveal nature of the protection, facilitate future researches on $\mathrm{AO}$ protection, and provide guidance for fabrication surface polymer
\end{abstract}

收稿日期: 2018-10-29; 收到修改稿日期：2018-11-29

基金项目：“十三五” 装备预研项目(170441422174); 国家自然科学基金青年项目(51802332); 上海市青年科技英才扬帆计 划项目(18YF1427100); 上海硅酸盐研究所创新项目(Y85ZC2120G, Y75ZC2120G)

Equipment Pre-research Foundation of China (170441422174); National Natural Science Foundation of China (51802332); Shanghai Sailing Program(18YF1427100); The Innovation Fund of Computational Materials Center from SICCAS (Y85ZC2120G, Y75ZC2120G)

作者简介: 李吴耕(1994-), 男, 博士研究生. E-mail: lihaogeng@student.sic.ac.cn

通讯作者: 章俞之, 研究员. E-mail: yzzhang@mail.sic.ac.cn; 宋力昕, 研究员. E-mail: lxsong@sunm.shcnc.ac.cn 
materials used in domestic parts of the aerospace craft, especially the large-scale flexible space solar cell array

Key words: atomic oxygen; polymer; space protection; surface modification; polyimide; computational simula-

tion; review

聚合物材料，诸如聚酰亚胺(Polyimide，PI)等, 是空间飞行器中主要的复合结构基体材料, 具有优 异的光、热、机械以及绝缘性能, 是制造航天器中 刚性或柔性太阳能电池基板不可替代的材料 ${ }^{[1-4]}$, 已广泛应用于各种航天设备与器件。但在高度为 200 700 km 的低地球轨道(Low Earth Orbit, LEO)空 间区域聚集着由太阳紫外线解离氧气分子所产生的 原子氧(Atomic Oxygen, AO), 当空间飞行器以第一 宇宙速度运行在 LEO 时, 相当于 AO 以 7 8 km/s 的相对速度撞击飞行器表面, 撞击平均动能达到 $5 \mathrm{eV}$, 且具有较强的氧化性 ${ }^{[5-6]}$, 会对表面的聚合物 材料造成不同程度的氧化剥蚀和质量损失。根据轨 道高度和太阳粒子活动的变化, $\mathrm{AO}$ 粒子束流通量通 常在 $10^{7} \sim 10^{13}$ atoms $\cdot \mathrm{cm}^{-2} \cdot \mathrm{s}^{-1}$ 之间, 最强时可达 $10^{15}$ atoms $\cdot \mathrm{cm}^{-2} \cdot \mathrm{s}^{-1[7-11]}$ 。在 $\mathrm{AO}$ 大通量、高能量的轰 击下, 聚合物材料原有的光、热以及机械性能会逐 渐衰退, 将严重影响在轨航天器的长期正常工作, 导致航天器的服役寿命大为缩短 ${ }^{[12-15]}$ 。

为应对 $\mathrm{AO}$ 的威胁, 国内外航天科研人员采用地 面模拟试验、空间飞行搭载试验 ${ }^{[16]}$, 以及计算模拟 等途径, 逐步开展了聚合物表面 AO 防护技术及其 作用机理研究。目前探索的两个主要方向是: 1)在聚 合物分子结构中引入特定元素所作的体材改性防护, 2)在聚合物材料表面制备 $\mathrm{AO}$ 防护涂层或改性防护 层所作的表面防护。这两种技术方向在长期低轨飞 行器(如空间实验室)表面的防护方面, 特别是太阳 能电池基板表面防护方面有各自的特点和优势。目 前, 随着我国航天战略的发展，长期在轨空间飞行 器的研究已经提上日程, 其太阳能电池基板表面聚 合物的 $\mathrm{AO}$ 防护工作也愈显重要。因而, 针对聚合 物材料表面 $\mathrm{AO}$ 进行防护技术研究, 深入探讨表面 防护技术与 $\mathrm{AO}$ 防护作用机理, 对研制我国大型柔 性空间太阳能电池基板和保障在轨空间飞行器的长 期正常服役都具有重要意义。

\section{1 聚合物表面 AO 防护}

\section{1 体材改性防护}

将特定元素基团或特定元素纳米颗粒引入聚合 物材料的分子结构, 使聚合物材料在遭受 $\mathrm{AO}$ 冲击 时在原位生成 $\mathrm{AO}$ 惰性层, 阻止 $\mathrm{AO}$ 对聚合物基体
造成进一步侵蚀, 这样的防护方法即为体材改性防 护。引入元素包括了 $\mathrm{P} 、 \mathrm{Zr} 、 \mathrm{Si}$ 等。

在含磷基团体材改性的研究中, 使用苯基氧化 膦(Phenylphosphine Oxide, PPO $)^{[17-18]}$ 基团体材改性 聚合物材料的 AO 防护性能最为突出。含有 PPO 基 团的聚合物材料在 AO 冲击下, 表面磷酸化作用程 度增强, 生成一层致密的聚磷酸酯网状结构, 对 $\mathrm{AO}$ 的氧化侵蚀有明显的抵抗作用。以 PI 为例, Wei 等 ${ }^{[19]}$ 合成了含有双 [4-(3-氨基苯氧基)苯基]苯基氧 化磷单体(mBAPPO)的 PI 前驱体, 通过随机共聚反 应将 mBAPPO 基团引入 PI 分子结构, 遭受 AO 冲 击后薄膜质量损失相比未改性 PI 降低了 $75 \%$ 左右, 侵蚀量相比下降 $60 \%$ 。虽然有 PPO 防护的 PI 表面 完整度优于未改性 PI 表面, 但是通过 SEM 观察到 裂纹状侵蚀痕迹, 在长期暴露过程中 $\mathrm{AO}$ 有可能通 过这些表面缺陷进一步向内部侵蚀, 进而导致防护 层防护性能的失效。

金属锆的氧化产物 $\mathrm{ZrO}_{2}$ 对 $\mathrm{AO}$ 侵蚀具有较高的 耐受性。以 PI 为例, Xiao 等 ${ }^{[20]}$ 在 PI 前驱体中添加 了正丁醇锆, 通过 Sol-Gel 法制备了含锆 PI 膜, AO 侵蚀后的质量损失与侵蚀率下降近一个数量级。Lü 等 ${ }^{[21]}$ 通过对 PI 与 $\mathrm{ZrO}_{2}$ 混合粉末的热压, 制备了含 有纳米 $\mathrm{ZrO}_{2}$ 粒子的 PI, 使改性后的 PI 薄膜兼具良 好的 $\mathrm{AO}$ 防护性能和耐磨性能。

由于 $\mathrm{Si}$ 的氧化产物是 $\mathrm{AO}$ 防护性能极高的 $\mathrm{SiO}_{x}$, 所以含 $\mathrm{Si}$ 体材改性防护的聚合物获得了较多关注, 其中具有代表性的改性基团是笼型倍半硅氧烷以及 聚硅氧烷。笼型倍半硅氧烷(Polyhedral Oligomeric Silsesquioxane, POSS)纳米基团呈现出有机无机杂 化结构, 其分子结构(图 1)内部是由硅树脂与氧组 成的 $\left(\mathrm{SiO}_{1.5}\right)_{x}$ 无机框架, 可以提升聚合物的 $\mathrm{AO}$ 防护 能力; 外部被多种功能化纳米化合物有机基团包裹, 有利于将 POSS 引入到聚合物的分子结构中 ${ }^{[22-24]}$ 。 此外, 面对 AO 的氧化剥蚀, POSS 还具有一定的损 伤自愈性 ${ }^{[25]}$ 。

美国空军实验室的 Tomczak 等 ${ }^{[26]}$ 和 Brunsvold 等 ${ }^{[27]}$ 通过合成含 POSS 的 PI 前驱体, 将侧基团为苯 胺的 POSS 引入到了 PI 的主链中, 并验证了 AO 的防 护能力。以色列的 Verker 等 ${ }^{[28-29]}$ 将三硅醇苯基 POSS 与 $\mathrm{PI}$ 前驱体进行复合, 研制了 PI/POSS 复合薄膜, $\mathrm{AO}$ 侵蚀后的质量损失相比下降 50\%左右, 但 SEM 


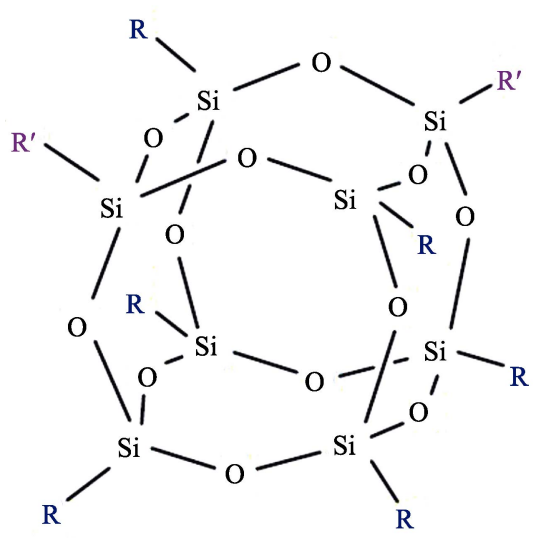

图 1 笼型倍半硅氧烷分子结构式(POSS) $)^{[24]}$

Fig. 1 Molecular structure of polyhedral oligomeric silsesquioxane (POSS) ${ }^{[24]}$

表征结果显示表面存在孔洞。国内研究人员 Fang 等 ${ }^{[30]}$ 以 PI 气凝胶为基体, 采用超临界二氧化碳干燥 工艺制备了 $\mathrm{PI} / \mathrm{POSS}$ 纳米复合气凝胶, $\mathrm{AO}$ 侵蚀率相 比下降 $80 \%$ 左右; Lei 等 $^{[31]}$ 合成了 POSS-二胺单体, 通过与酰亚胺共聚，制备了支链含有 POSS 的 PI 薄 膜, 遭受 $\mathrm{AO}$ 冲击后表面粗䊁度以及质量损失明显 下降; Liu 等 ${ }^{[32]}$ 将合成的氟丙基-POSS 单体引入偏 二氟乙烯(PVDF)中，有效提升了 PVDF 对 AO 的防 护能力。使用聚硅氧烷对 PI 进行体材改性, 同样具 有较好的防护效果与自愈合能力。Lei 等 ${ }^{[33]}$ 将高度 拓扑超支化的聚硅氧烷编入 PI 骨架, 在不影响 PI 自身光学性能的条件下增加了 $\mathrm{Si}$ 元素含量 $(29.7 \mathrm{wt} \%)$, 侵蚀率相比降低到了 $7.97 \%$, 且表面更加光滑平整。

体材改性防护的优势在于, 采用化学合成的方 式将防护基团引入聚合物基体的分子结构，防护层 与聚合物基底之间为强结合力的化学键合, 且膜层 成分一致可以避免应力, 不会在存储、运输以及使 用过程中开裂、脱落。但是, 外部基团的引入会对 聚合物薄膜原有的力学性能产生负面影响, 产生提 升聚合物 $\mathrm{AO}$ 防护能力与维持聚合物原有性能之间 的矛盾: 提高 $\mathrm{AO}$ 防护基团含量可能导致聚合物原 有性能下降; 维持聚合物的原有性能则使得 AO 防 护基团含量偏低, 难以形成致密且连续的 $\mathrm{AO}$ 防护 结构。因此, 通过进一步改进改性方法, 或者寻找新 的防护基团, 在不影响聚合物基体原有性质的前提 下提升聚合物材料的 $\mathrm{AO}$ 防护能力, 是体材改性防 护的发展趋势与目标。

\section{2 表面防护}

聚合物的表面防护是另一种常用的 AO 防护方 法，分为防护涂层以及有机/无机复合改性层两类, 研究时间较长, 技术相对成熟。

\subsection{1防护涂层}

根据涂层材料的组成与性质，聚合物材料表面 $\mathrm{AO}$ 防护涂层分为无机涂层与有机涂层, 通常采用 Sol-Gel、磁控溅射、气相/液相沉积等物理法制备在 聚合物表面。基于无机氧化物或金属氧化产物自身 对 $\mathrm{AO}$ 的惰性, 无机涂层在聚合物表面 $\mathrm{AO}$ 防护的 研究中获得了较早关注。常用的无机涂层包括了 $\mathrm{SiO}_{2}{ }^{[34]} 、 \mathrm{Al}_{2} \mathrm{O}_{3}{ }^{[35]} 、 \mathrm{TiO}_{2}{ }^{[36]} 、 \mathrm{MgO}^{[37-38]} 、 \mathrm{~V}_{2} \mathrm{O}_{5}{ }^{[39]} 、$ $\mathrm{ZnO}^{[40]} 、 \mathrm{SnO}_{2}{ }^{[41]}$ 、氧化铟锡 $(\mathrm{ITO})^{[42]}$, 以及 $\mathrm{Si} / \mathrm{Al}^{[43]}$ 、 $\mathrm{ZnO} / \mathrm{Al}^{[44]}$ 等多种金属氧化物组成的复合涂层。聚合 物表面无机涂层遭受 $\mathrm{AO}$ 冲击后可以保持表面光滑 平整, 质量损失相比下降两个数量级, $\mathrm{AO}$ 防护性能 十分出色。但是, 无机材料自身柔韧性差, 在运输过 程中容易出现裂纹, 并且难以使用在弯曲表面上。 此外, 无机涂层与聚合物基底的热膨胀系数不匹配, 在面对 $\mathrm{AO}$ 与紫外辐照(Ultraviolet, UV)、冷热交变 等空间环境的协同作用时容易发生开裂、脱落的状 况。根据目前的研究进展可知, AO 对于表面缺陷十 分敏感，会通过防护涂层的缺陷处向基体内部进行 掏蚀，造成聚合物基体严重损伤。因此，增加无机涂 层的柔韧性以及改善无机涂层与聚合物基体的结合 性, 使无机涂层拥有防脱落防开裂功能, 是无机防 护涂层目前的研究方向。

有机防护涂层通常选择聚硅氧烷、聚硅铝氮烷、 聚氟化物以及聚硅氮烷等作为防护基团 ${ }^{[45-46]}$, 其中, 聚硅氮烷在热分解环境下可以转变成为对 $\mathrm{AO}$ 具 有较好防护能力的 $\mathrm{SiN} / \mathrm{SiC}$, 受到了较多关注。Duo 等 ${ }^{[47-48]}$ 通过浸渍法在 PI 表面制备聚硅氮烷、聚硅氮 烷/POSS 复合有机涂层, 遭受 AO 冲击后表面依然 保持光滑, 质量损失下降一个数量级, 且表面没有 出现裂痕。有机防护涂层相较于无机涂层拥有更好 的柔韧性, 与聚合物基体的结合性也要优于无机涂 层，但是受限于有机物自身性质，面对空间环境的 紫外辐照出现老化现象。

\subsection{2 有机/无机复合改性层}

根据上述研究可以发现, 无机涂层的 $\mathrm{AO}$ 防护 性能优异, 但是与基底结合性差且缺乏柔韧性; 有 机涂层具有一定柔韧性且与基底结合性良好，但 $\mathrm{AO}$ 防护能力却不够出众。为了结合有机/无机防护 各自的优点, 国内外研究者使用表面化学改性的方 法，制备出了与聚合物基底结合性良好且 $\mathrm{AO}$ 防护 性能优异的有机/无机复合改性层。以 PI 的有机/无 机复合硅改性层的制备为例 ${ }^{[99]}$, 使用诸如 UV 辐照 氧化、等离子体氧化、火焰处理、离子轰击以及湿 化学(图 2)等方法对 PI 进行表面活化改性, 增加 PI 


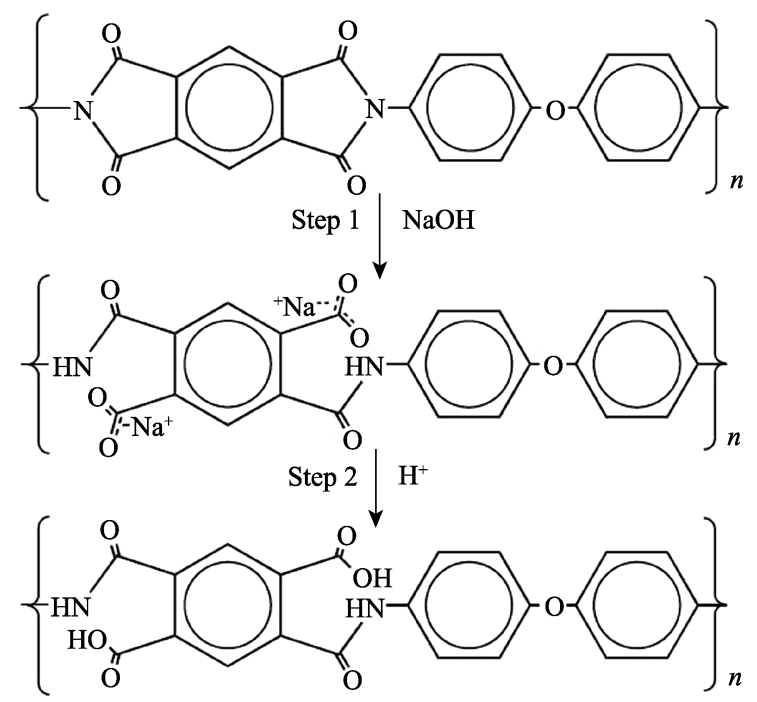

图 2 PI 表面改性生成聚酰胺酸示意图 ${ }^{[50]}$

Fig. 2 PI surface modification to form polyamic acid: $\mathrm{NaOH}$ hydrolysis of PI (Step 1) to form sodium salt of polyamic acid followed by acidification (Step 2 ) to form polyamic acid ${ }^{[50]}$

表面含氢活性功能团(如羟基)的含量，通过取代反 应将有机硅基团植入 PI 表面, 经表面稳定化处理后 形成渐变的有机/无机复合改性层，在克服了无机涂 层自身脆性以及与基底的热膨胀系数不匹配等缺点 的同时，提供了优异的 $\mathrm{AO}$ 防护性能。

加拿大 Integrity Testing Laboratory 公司的 Kleiman 等于九十年代中期研发了 Photosil ${ }^{[51]}$ 紫外辐照光活 化技术以及 Implantox ${ }^{[50]}$ 离子注入表面改性技术, 对 PI 表面分别采取化学改性以及物理改性, 将 AO 的侵蚀率降低了 1 2 个数量级, 被广泛地应用在 NASA 空间飞行试验中。根据表面 XPS 分析结果 ${ }^{[52]}$, Photosil 的硅烷化深度达到了 $0.5 \mu \mathrm{m}$, 改性层与PI 基体 的结合十分牢固; 根据 SEM 照片 ${ }^{[52-53]}$ (图 3)可以看 出, 经过 Photosil 法和 Implantox 法处理后的样品相 比于未处理样品, 遭受 $\mathrm{AO}$ 冲击后表面没有明显侵 蚀伤痕, 与未被冲击的原始表面相比几乎没有差别,
$\mathrm{AO}$ 防护效果出众。但是，光活化技术存在光源与基 底距离不同导致的 line-of-sight效应，不适用于异型 复杂基底; 离子注入改性虽然能改善聚合物的 $\mathrm{AO}$ 防护能力，但是离子注入改性易导致表面碳化且成 本高，不易于工艺控制和生产制造 ${ }^{[54]}$ 。

国内研究人员普遍使用湿化学法对 PI 进行表 面活化改性。使用一定浓度的碱液进行表面化学改 性，可以去除 PI 表面的钝化层，同时增加表面羟基 含量 ${ }^{[55]}$; 使用硅烷偶联剂辅助表面改性硅烷化, 可 以改善改性层与聚合物基体的界面状态。 Liu 等 ${ }^{[56]}$ 使用不同种类的硅烷偶联剂, 分别在酸性和碱性环 境下对 PI 进行表面改性, 发现在酸性环境下使用硅 烷偶联剂 APTES 进行表面改性, 与其他硅烷偶联 剂相比具有更好的 $\mathrm{AO}$ 防护性能。王丹等 ${ }^{[57]}$ 分别使 用 $\mathrm{NaOH}$ 水热、硅烷偶联剂 KH-550 溶剂热以及两 者的组合对 PI 进行表面化学改性, 根据接触角测试 试验发现 $\mathrm{NaOH}$ 水热/KH-550 溶剂组合的改性效果 最好, 改性后表面使用提拉法镀覆 $\mathrm{SiO}_{2}$ 防护层(图 4 , 表 1), 防护效果十分出色。Gu 等 ${ }^{[54]}$ 采用湿法活 化硅烷化的改性方式对 PI进行表面改性, 对 PI 原有 的光学、力学性能影响较小, 改性后 PI 的 AO 防护 性能提高了 2 个数量级以上。

PI 在表面硅烷化后, 新生成的表面结构中存在 大量碳氢化合物基团，与 $\mathrm{AO}$ 的反应中有助于硅氧 基团的生成，间接提升了聚合物材料的 $\mathrm{AO}$ 防护能 力 ${ }^{[49]}$ 。但是在实际的空间环境中, 这些碳氢化合物 的氧化过程会为表面带来额外污染 ${ }^{[58]}$ 。为此, 复合 改性防护层在进入空间前需要进行表面稳定化处理, 排除额外污染的同时, 使表面改性层提前形成稳定 的 $\mathrm{AO}$ 防护结构 ${ }^{[49]}$ 。Xie 等 ${ }^{[59]}$ 通过 Sol-Gel 法制备 $\mathrm{Si} / \mathrm{Ti}$ 复合改性层, 经过稳定氧化热处理后, 在冷热 交变以及 UV 辐照等测试后未有开裂现象, 质量损 失下降 1 2 个数量级。但是 Sol-Gel 法在表面缺陷 以及密度控制上存在一定问题。
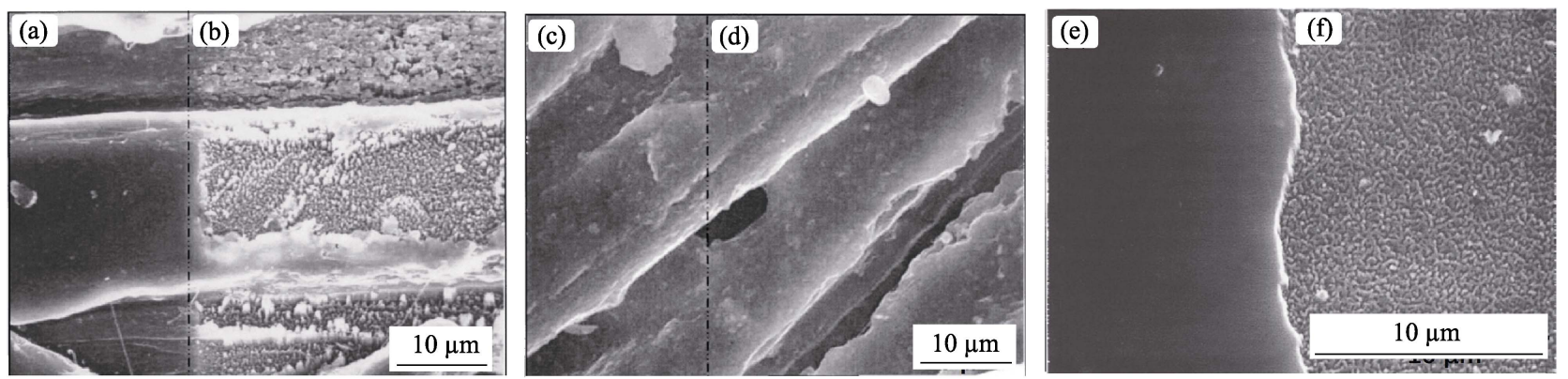

图 $3 \mathrm{AO}$ 轰击(有效积累通量约为 $2.0 \times 10^{20} \mathrm{atoms} / \mathrm{cm}^{2}$ )下的样品 SEM 照片 ${ }^{[52-53]}$, 未经处理的(a)被遮挡区域及(b)暴露区域; Photosil $^{\mathrm{TM}}$ 处理后的(c)被遮挡区域及(d)暴露区域，和暴露的(e) Implantox 处理后的区域以及(f)无防护处理区域

Fig. 3 SEM images of AO exposed (effective fluence $\sim 2.0 \times 10^{20}$ atoms $/ \mathrm{cm}^{2}$ ) samples: untreated samples ${ }^{[52-53]}$

(a) masked section and (b) exposed section, images of Implantox ${ }^{\mathrm{TM}}$ treatment being (c) masked and (d) exposed sections, and AO exposed (e) Implantox-treated section and (f) untreated sections 
表 1 AO 对 PI 样品的侵蚀量 ${ }^{[56]}$

Table 1 Erosion yield of atomic oxygen on PI samples ${ }^{[56]}$

\begin{tabular}{lccccc}
\hline \multicolumn{1}{c}{ Sample } & $F /\left(\times 10^{20} \mathrm{O}\right.$ atom $\left.\cdot \mathrm{cm}^{-2}\right)$ & $\Delta M / \mathrm{mg}$ & $A / \mathrm{cm}^{2}$ & $(\Delta M / A) /\left(\mathrm{mg} \cdot \mathrm{cm}^{-2}\right)$ & $E /\left(\times 10^{-24}, \mathrm{~cm}^{3} \cdot \mathrm{atom}^{-1}\right)$ \\
\hline Kapton & 3.09 & 5.03 & 3.14 & 1.60 & 3.65 \\
$\mathrm{SiO}_{x}$ coated PI & 3.09 & 0.17 & 3.14 & 0.05 & 0.12 \\
\hline
\end{tabular}
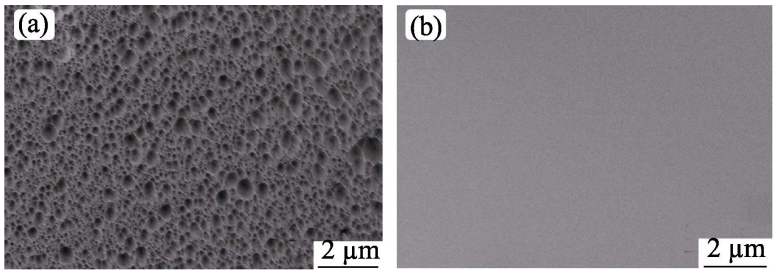

图 4 暴露在原子氧辐照后的样品表面形貌 ${ }^{[56]}$ : (a)无防护处 理的原始 PI 和(b)镀覆 $\mathrm{SiO}_{2}$ 防护层的 PI

Fig. 4 SEM images of AO exposed (effective fluence $2.0 \times 10^{20}$ atoms $\left./ \mathrm{cm}^{2}\right)$ sample surfaces morphology ${ }^{[56]}$ : untreated original PI and (b) $\mathrm{SiO}_{2}$ coated PI

总体来说，聚合物表面有机/无机复合改性防护 层结合了体材改性和一般防护涂层的优点，与基底 结合性良好且有一定柔性, 具有较好的综合防护性 能。但是，目前复合体系内各组分之间与 AO 协同 作用防护机理尚不清楚, 缺乏复合防护体系与 $\mathrm{AO}$ 作用机理的研究。同时, 由于作用机理的缺乏，目前 没有建立适用于复合防护体系的 $\mathrm{AO}$ 防护性能评估 标准，仅采用空间搭载试验或地面模拟试验对种类 繁多的复合防护体系进行对比和篮选, 存在试验周 期长, 试验成本高的缺陷。除此之外，常规试验结合 材料表征测试的研究方法对作用机理在微尺度的分 析存在一定的困难, 需要借助其他研究方法辅助作 用机理的分析和研究。

\section{AO 作用的计算模拟}

$\mathrm{AO}$ 作用的计算模拟借助理论与算法, 或使用 计算模拟软件, 构建 $\mathrm{AO}$ 作用的计算模型, 对轰击 方式、损伤效果、防护反应以及反应产物等内容进 行计算模拟。依据不同理论, $\mathrm{AO}$ 作用的计算模拟可 以分为理论数值计算模拟、反应分子动力学计算模 拟，以及基于第一性原理的计算模拟。不同于空间 搭载试验和地面模拟试验, $\mathrm{AO}$ 作用的计算模拟从基 础理论出发, 模拟 $\mathrm{AO}$ 的侵蚀作用以及不同防护体 系的防护作用, 并依据试验数据对模型进行完善与 验证, 对 AO 的防护作用机理的研究具有一定的优 势。但是, 由于 $\mathrm{AO}$ 的侵蚀过程极为复杂, 涉及的科 学问题繁多, 通过有限理论构建计算模拟模型难以同 时涵盖所有的宏观或微观作用过程与细节, 导致计算 模拟结果难以对 $\mathrm{AO}$ 防护作用机理进行整体解释。

\section{1 理论数值计算模拟}

在早期的数值模拟理论模型中, NASA ${ }^{[60]}$ 结合 地面模拟试验与空间飞行试验得到的数据建立了基 于 Monte Carlo 算法的二维掏蚀模型。该模型可以 模拟出 $\mathrm{AO}$ 对聚合物基底的二维纵向掏蚀形状, 在 某些参数设置下与试验结果拟合较好。国内外研究 人员基于 Monte Carlo 算法建立掏蚀模型对不同防 护涂层的研究不在少数 ${ }^{[61-64]}$, 并根据试验数据对模 型进行不断的完善。

除了基于 Monte Carlo 算法的掏蚀模型外, 自 上世纪九十年代开始, 国外理论研究者根据已有的 试验数据，依据不同理论和假设建立了反应性散射 模型、键合方向性模型，以及量子力学模型等强烈 依赖于经验的理论模型 ${ }^{[65]}$, 可以与试验结果产生不 同程度的拟合。国内的陈来文等 ${ }^{[66]}$ 建立了分子随机 动力学模型以及普适性较强的自洽理论模型, 数值 模拟结果与 STS-46 飞行试验数据相近。上述数值模 拟模型对于 AO 表面侵蚀的研究重点各有不同, 但 仅仅考虑 $\mathrm{AO}$ 与聚合物材料表面的相互作用, 与其 他空间环境威胁作用(紫外辐照、UV、等离子体)的 协同效应也需要纳入到模型当中。 $\mathrm{Liu}$ 等 ${ }^{[67]}$ 建立了 热控涂层遭受 $\mathrm{AO}$ 与 UV 侵蚀的分析模型, 拟合效 果与三颗国内低轨气相卫星的数据进行对比, 整体 效果较好, 但是该模型的完善需要对更多的空间试 验数据来完善模型。陈来文等 ${ }^{[68]}$ 创立了针对含氢聚 合物的非支链式反应模型, 推导出了聚合物材料质 量变化的统一等式, 通过给定 $\mathrm{AO}$ 的积分通量与流 量, 可以推导出等效暴露时间以及质量损失。

\section{2 反应分子动力学计算模拟}

基于牛顿力学的分子动力学 (Molecular Dynamic, MD)计算模拟适用于 $\mathrm{AO}$ 与防护体系在原子 尺度的作用过程计算模拟。相比于理论模型的符号 与公式, MD 计算模拟凭借计算软件的计算能力以 及交互性, 对研究对象的动态特性描述更加直观具 体, 可以更好地结合试验对防护作用机理进行解 释。Van Duin 等 ${ }^{[69]}$ 建立的反应力场(Reactive Force Field, ReaxFF), 依据键长与键级以及键级与键能之 间的整体关系进行成键与断键的判定，是大分子量 反应体系的 MD 计算模拟中最常用的计算力场。由 此, 反应分子动力学计算模拟十分适用于计算模拟 
$\mathrm{AO}$ 与聚合物材料之间在原子尺度的反应过程。除 此之外, 使用 ReaxFF 力场的反应分子动力学模拟, 可以获得在极短时间区间内可能存在的微观变化与 反应产物, 弥补了使用常规试验测试方法分析反应 机理时在时间与空间尺度上的不足。

Van Duin 等 ${ }^{\left[{ }^{[0]}\right.}$ 使用反应分子动力学在大尺寸体 系条件下模拟了原始 PI、POSS/PI、非晶硅以及聚 四氟乙烯经过 $\mathrm{AO}$ 冲击的反应以及崩解过程。通过 构建原子数在 2000 个以上的上述分子模型集合体 系, 选择 $\mathrm{NVT}$ 系综在 $300 \mathrm{~K}$ 条件下模拟 $\mathrm{AO}$ 的撞击 过程, 并且通过对比不同防护体系的温度变化、质 量变化以及侵蚀产物的区别, 获得不同基团与 $\mathrm{AO}$ 的反应规律。Rahmani 等 ${ }^{[71]}$ 同样建立了较为庞大的 模拟体系(240 以上分子个数, 图 5), 借助 ReaxFF 力 场分析了 POSS、石墨稀(Graphene, Gr)、碳纳米管 (CNTs) 以及上述物质在不同排列方式下对 $\mathrm{AO}$ 冲击 侵蚀的防护作用。Zheng 等 ${ }^{[72]}$ 使用反应分子动力学 分析对比了聚偏氟乙烯(PVDF)、三氟丙基-POSS 化 合物(FP-POSS)以及两者混合的三种材料, 经过 AO 冲击的崩解过程模拟, 其模拟数据与 NASA 的 MISSE2 空间飞行试验相近。Li 等 ${ }^{[25]}$ 根据地面模拟 试验探究了 PI 薄膜的 POSS 掺杂含量对 AO 防护能 力的影响, 并根据 ReaxFF MD 计算模拟得到的微观 损伤深度变化对试验结果进行了分析与解释。相较 于地面 $\mathrm{AO}$ 模拟试验以及空间飞行试验, 采用反应 分子动力学计算模拟对不同防护基团与 $\mathrm{AO}$ 的反应 过程进行探究, 可以节省较多的时间与成本, 并得 到较为可靠的数据, 为试验结果提供有效参考。

\section{3 基于第一性原理的计算模拟}

基于第一性原理的计算模拟不同于使用经典力 学体系的计算模拟, 应用量子力学的方法分析体系
内的原子核与电子相互作用, 可以获得 $\mathrm{AO}$ 防护体 系内的能量、态密度以及原子位置变化等内容, 得 到诸如氧化反应路径、原子氧扩散能垒、原子运动、 键合反应能量等数据。Gindulyte 等 ${ }^{[73]}$ 使用 ab initio 分子轨道计算, 研究并证明了单个 $\mathrm{O}\left({ }^{3} \mathrm{P}\right)$ 冲击可以 导致聚乙烯产生链式断裂。对于 $\mathrm{Gr}$ 的 AO 防护应用 的研究, Zhang 等 ${ }^{[74]}$ 使用密度泛函(Density Functional Theory, DFT)的计算模拟方法计算了 AO 面对 本征 $\mathrm{Gr} / \mathrm{Gr} 8$ 环 $/ 10$ 环/12 环缺陷, 以及上述缺陷组合 的扩散能垒, 并与 $\mathrm{AO} 5 \mathrm{eV}$ 冲击能量进行比较, 获 得不同 $\mathrm{Gr}$ 对于 $\mathrm{AO}$ 向内扩散的抑制作用, 并与地面 $\mathrm{AO}$ 试验进行对比, 在得到验证的同时为 $\mathrm{Gr}$ 的原子 氧防护能力提供了解释。 $\mathrm{Gu}^{[54]}$ 利用 DFT 理论计算 模拟了 PI 表面硅烷化的化学反应机制, 结合 PI 表面 湿法活化硅烷化的改性研究, 揭示了 PI 表面硅烷化 以及催化亚胺化的机理。计算模拟的方法可以对试 验结果进行理论验证, 同时对试验得到的定性规律 进行理论解释。

基于第一性原理的计算模拟可以在量子尺度针 对诸如电子态、能量以及原子运动等特性进行精确 计算, 并结合试验, 对 $\mathrm{AO}$ 作用过程中的反应、扩 散、成键等问题进行分析。虽然计算的成本较高, 构 建直观且普适性的模型难度较大, 但是可以从量子 力学角度反映 $\mathrm{AO}$ 与防护涂层或基团的作用特性, 为试验作用规律的诠释提供更多角度, 在作用机理 分析上有较大的潜力。

\section{3 展望}

$\mathrm{AO}$ 是空间太阳能电池基板的主要空间环境威 胁, 严重影响了太阳能电池基板的可靠性。研究发
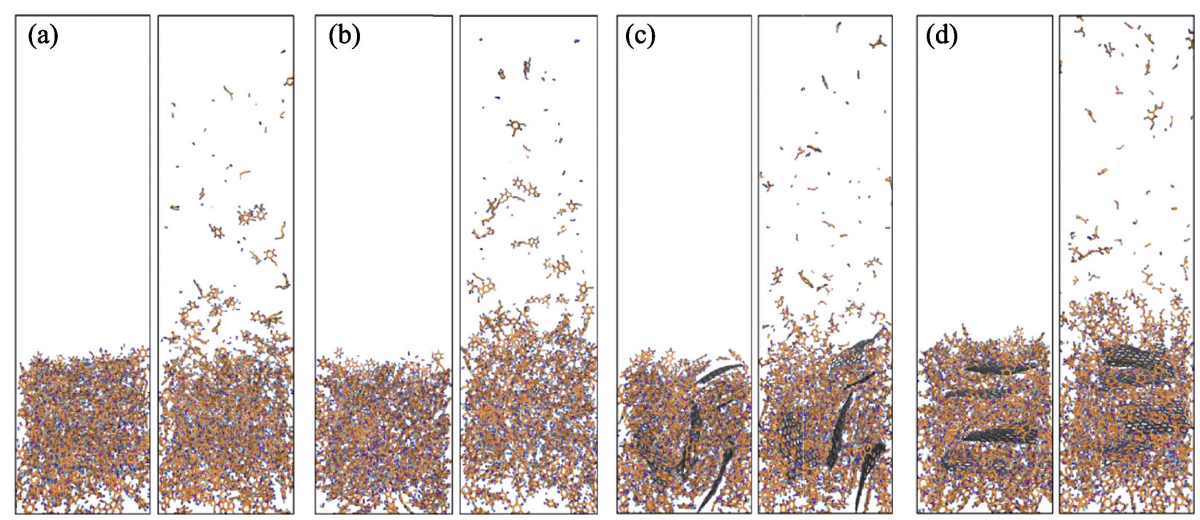

图 5 不同 PI 防护体系遭受 $\mathrm{AO}$ 轰击前(左侧, $t=0$ )和轰击后(右侧, $t=35 \mathrm{ps}$ )的计算模拟图像 ${ }^{[71]}$ :

PI 枝接(a) $15 \mathrm{wt} \%$ POSS 和(b) $30 \mathrm{wt} \%$ POSS; $15 \mathrm{wt} \%$ 石墨稀采用(c)随机取向和(d)定向排列

Fig. 5 Initial (left, $t=0$ ) and final (right, $t=35 \mathrm{ps}$ ) simulation snapshots of different PI protection system under AO impact ${ }^{[71]}$ : PI-grafted (a) $15 \mathrm{wt} \%$ POSS and (b) $30 \mathrm{wt} \%$ POSS; $15 \mathrm{wt} \%$ graphene (c) randomly oriented and (d) aligned 


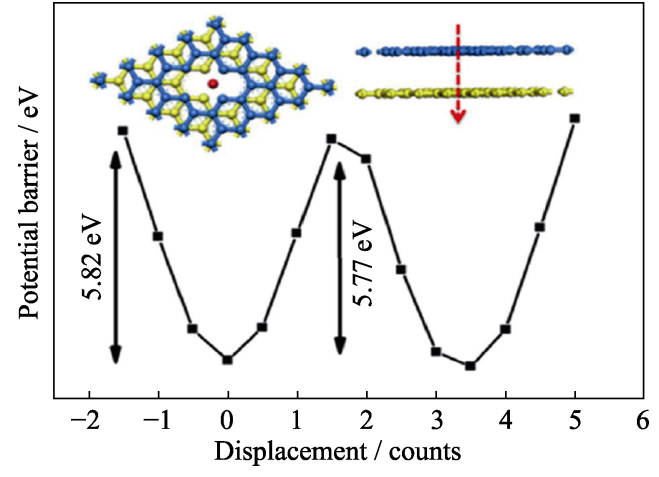

图 6 氧原子通过匹配的双空位缺陷渗透进入双层石墨烯隔 层的 DFT 势垒计算 ${ }^{[74]}$

Fig. 6 Potential barrier of an $\mathrm{O}$ atom permeating into interlayer of double-layer graphene with matched divacancy defect ${ }^{[74]}$

展太阳能电池基板表面聚合物材料的 $\mathrm{AO}$ 防护技术, 为长寿命空间航天器的正常运作提供了保障。目前 对这些防护技术以及作用机理的研究中，仍然存在 一些问题需要进一步加以研究:

1)对于体材改性防护，防护基团含量越高，防 护效果越好，但是防护基团在聚合物中的占比过高 会影响到聚合物自身性能。因此采用体材改性的思 路进行 AO 防护材料研究时, 需要注意防护基团的 最优含量以及分散性问题, 在不影响聚合物本身性 能的前提下均匀地分散在聚合物表面，为聚合物基 体提供防护。

2)对于表面防护，注重无机涂层与聚合物基体 的结合性以及涂层自身的柔韧性，可以提高无机涂 层的实用性。有机/无机复合改性防护层在结合有机 无机防护技术各自优势的条件下，优化复合体系、 制备工艺和改性方法, 也可以开展新体系与新工艺 的研究, 进一步提升复合改性层的综合防护性能。

3)计算模拟在 $\mathrm{AO}$ 作用机理的研究中具有较大 的潜力, 然而计算模型的设计难以避免具有一定的 理想性和微观性, 因此, 根据试验数据构建计算模 型, 使用计算结果辅助分析试验结果, 结合计算模 拟与试验开展研究是未来的发展方向。

4)AO 的防护技术种类多，防护作用机理涉及 多种理论与体系, 使用经典力学在分子尺度构建普 适性的防护理论框架或是作用模型难度较大, 建立 普适性的微观计算模型更是难上加难。因此, 在对 作用机理展开研究时, 可以将整个 AO 作用过程化 整为零, 发挥计算模拟在微尺度分析方面的优势, 结合试验对特定科学问题进行诠释, 再将不同问 题的分析结果进行整合, 总结分析出完整的作用 机理。

\section{参考文献:}

[1] DEVER J A, MILLER S K, SECHKAR E A, et al. Space environment exposure of polymer films on the materials international space station experiment: results from MISSE 1 and MISSE 2. High Perform. Polym., 2008, 20(4/5): 371-387.

[2] ZHANG W, YI M, SHEN Z G, et al. Protection against atomic oxygen erosion of oxide coatings for spacecraft materials. Journal of Beijing University of Aeronautics and Astronautics, 2013, 39(8): 1074-1078.

[3] LEI X F. Functional Polyimide/silicon Films: Fabrication and Properties. Xi'an: Northwestern Polytechnical University PhD thesis, 2016.

[4] LIAW D J, WANG K L, HUANG Y C, et al. Advanced polyimide materials: syntheses, physical properties and applications. Prog. Polym. Sci., 2012, 37(7): 907-974.

[5] XIONG Y Q, XIE S P. Measurement methods of atomic oxygen concentration. Journal of Transducer Technology, 1999, 18(3): $8-12$.

[6] SEMONIN D M, BRUNSVOLD A L, MINTON T K. Erosion of Kapton $H \circledR$ by hyperthermal atomic oxygen. J. Spacer. Rockets, 2006, 43(2): 421-425.

[7] SONG M M. Study on Erosion Effect of Atomic Oygen on Polyimide and Its Protective Technology in LEO Environment. Nanchang: Jiangxi Science and Technology Normal University Master Thesis, 2012.

[8] WANG C B. Study on the Degradation Behavior of Organic/Inorganic Protective Materials in Atomic Oxygen Environment. Changchun: Jilin University Master Thesis, 2017.

[9] SILVERMAN E M. NASA Contrator Report 4661, Part 1. Space Environmental Effects on Spacecraft: LEO Materials Selection Guide, part 1, N96-10860, Virginia: NASA, 1995.

[10] TIAN C, CHENG L F, LUAN X G. Degradation behaviour of C/C composites by atomic oxygen irradiation. Journal of Inorganic Materials, 2013, 28(8): 853-858.

[11] HOOSHANGI Z, FEGHHI S A H, SAEEDZADEH R. The effects of low earth orbit atomic oxygen on the properties of polytetrafluoroethylene. Acta Astronaut., 2016, 119: 233-240.

[12] DE GROH K K, BANKS B A. Atomic-oxygen undercutting of long duration exposure facility atomized-Kapton multilayer insulation. J. Spacer. Rockets, 1994, 31(4): 656-664.

[13] SHIMAMURA H, NAKAMURA T. Mechanical properties degradation of polyimide films irradiated by atomic oxygen. Polym. Degrad. Stabil., 2009, 94(9): 1389-1396.

[14] DE GROH K K, BANKS B A, MITCHELL G G, et al. NASA STI Pprogram. MISSE 6 Stressed Polymers Experiment Atomic Oxygen Erosion Data, NASA/TM-2013-217847, Ohio: NASA, 2013.

[15] BANKS B A, DILL G C, LOFTUS R J, et al. NASA STI Program. Comparison of Hyperthermal Ground Laboratory Atomic Oxygen Erosion Yields with Those in Low Earth Orbit, NASA/TM2013-216613, Ohio: NASA, 2013.

[16] BANKS B A, MILLER S K. NASA STI Program. Effects of Sample Holder Rdge Geometry on Atomic Oxygen Erosion Yield of Pyrolytic Graphite Exposed in Low Earth Orbit, NASA/TM2018-219910, Ohio: NASA, 2018.

[17] QING F L, CAO W Z. Mechanical Property Improvement of Novel AO Resistance PI Thin Films and the synthesis of Wide Width Films. China Space Science Society Space Materials Specialized Committee 2009 Academic Exchange Proceedings, 2009.

[18] JI H W. Synthesis and Atomic Oxygen Erosion Resistance Property of PPO-containing Polyimide Films. Changchun: Jilin University 
Master Thesis, 2014.

[19] WEI J H, GANG Z X, MING L Q, et al. Atomic oxygen resistant phosphorus-containing copolyimides derived from bis [4-(3-aminophenoxy) phenyl] phenylphosphine oxide. Sci. Ser. B, 2014, 56(6): 788-798.

[20] XIAO F, WANG K, ZHAN M. Atomic oxygen erosion resistance of polyimide $/ \mathrm{ZrO}_{2}$ hybrid films. Appl. Surf. Sci., 2010, 256(24): 7384-7388.

[21] LÜ M, WANG Q, WANG T, et al. Effects of atomic oxygen exposure on the tribological performance of $\mathrm{ZrO}_{2}$-reinforced polyimide nanocomposites for low earth orbit space applications. Compos. Pt. B-Eng., 2015, 77: 215-222.

[22] LI G, WANG L, NI H, et al. Polyhedral oligomeric silsesquioxane (POSS) polymers and copolymers: a review. J. Inorg. Organomet. Polym., 2001, 11(3): 123-154.

[23] MINTON T K, WRIGHT M E, TOMCZAK S J, et al. Atomic oxygen effects on POSS polyimides in low earth orbit. ACS Appl. Mater. Interfaces, 2012, 4(2): 492-502.

[24] LEI X F, QIAO M T, TIAN L D, et al. Improved space survivability of polyhedral oligomeric silsesquioxane (POSS) polyimides fabricated via novel POSS-diamine. Corros. Sci., 2015, 90: 223-238.

[25] LI X, AL-OSTAZ A, JARADAT M, et al. Substantially enhanced durability of polyhedral oligomeric silsequioxane-polyimide nanocomposites against atomic oxygen erosion. Eur. Polym. J., 2017, 92: 233-249.

[26] TOMCZAK S J, MARCHANT D, SVEIDA S, et al. Properties and Improved Space Survivability of POSS (Polyhedral Oligomeric Silsesquioxane) Polyimides. MRS Online Proc. Libr., 2004: 851.

[27] BRUNSVOLD A L, MINTON T K, GOUZMAN I, et al. An investigation of the resistance of polyhedral oligomeric silsesquioxane polyimide to atomic-oxygen attack. High Perform. Polym., 2004, 16(2): 303-318.

[28] VERKER R, GROSSMAN E, GOUZMAN I, et al. POSS-polyimide nanocomposite films: simulated hypervelocity space debris and atomic oxygen effects. High Perform. Polym., 2008, 20(4/5): 475-491.

[29] VERKER R, GROSSMAN E, ELIAZ N. Erosion of POSS-polyimide films under hypervelocity impact and atomic oxygen: the role of mechanical properties at elevated temperatures. Acta Mater., 2009, 57(4): 1112-1119.

[30] FANG G, LI H, LIU J, et al. Intrinsically atomic-oxygen-resistant POSS-containing polyimide aerogels: synthesis and characterization. Chem. Lett., 2015, 44(8): 1083-1085.

[31] LEI X, QIAO M, TIAN L, et al. Evolution of surface chemistry and morphology of hyperbranched polysiloxane polyimides in simulated atomic oxygen environment. Corros. Sci., 2015, 98: $560-572$.

[32] LIU Y Z, SUN Y, ZENG F L, et al. Characterization and analysis on atomic oxygen resistance of POSS/PVDF composites. Appl. Surf. Sci., 2014, 320: 908-913.

[33] LEI X F, CHEN Y, ZHANG H P, et al. Space survivable polyimides with excellent optical transparency and self-healing properties derived from hyperbranched polysiloxane. ACS Appl. Mater. Interfaces, 2013, 5(20): 10207-10220.

[34] DUO S W, SONG M M, LIU T Z, et al. $\mathrm{SiO}_{2}$ Coatings Prepared by Sol-Gel Process Protecting Silver from Atomic Oxygen Erosion. Applied Mechanics and Materials. Switzerland: Trans Tech Publications, 2012: 3044-3047.

[35] HEIMANN R B, KLEIMAN J I, LITOCSKY E, et al. High-pressure cold gas dynamic (CGD)-sprayed alumina-reinforced aluminum coatings for potential application as space construction material.
Surf. Coat. Technol., 2014, 252: 113-119.

[36] GOUZMAN I, GIRSHEVITZ O, GROSSMAN E, et al. Thin film oxide barrier layers: protection of Kapton from space environment by liquid phase deposition of titanium oxide. ACS Appl. Mater. Interfaces, 2010, 2(7): 1835-1843.

[37] QI H, QIAN Y, XU J, et al. Studies on atomic oxygen erosion resistance of deposited Mg-alloy coating on Kapton. Corros. Sci., 2017, 124: 56-62.

[38] QI H, QIAN Y, XU J, et al. An AZ31 magnesium alloy coating for protecting polyimide from erosion-corrosion by atomic oxygen. Corros. Sci., 2018, 138: 170-177.

[39] ERDOĞAN S, KÖYTEPE S, SECKIN T, et al. $\mathrm{V}_{2} \mathrm{O}_{5}$-polyimide hybrid material: synthesis, characterization, and sulfur removal properties in fuels. Clean Technol. Environ. Policy, 2014, 16(3): 619-628.

[40] CHEN L, LIU L, DU Y, et al. Processing and characterization of $\mathrm{ZnO}$ nanowire-grown $\mathrm{PBO}$ fibers with simultaneously enhanced interfacial and atomic oxygen resistance properties. RSC Adv., 2014, 4(104): 59869-59876.

[41] GOTLIB-VAINSTEIN K, GOUZMAN I, GIRSHEVITZ O, et al. Liquid phase deposition of a space-durable, antistatic $\mathrm{SnO}_{2}$ coating on Kapton. ACS Appl. Mater. Interfaces, 2015, 7(6): 3539-3546.

[42] OUYANG Q, WANG W, FU Q, et al. Atomic oxygen irradiation resistance of transparent conductive oxide thin films. Thin Solid Films, 2017, 623: 31-39.

[43] HUANG Y, LÜ S, TIAN X, et al. Interface analysis of inorganic films on polyimide with atomic oxygen exposure. Surf. Coat. Technol., 2013, 216: 121-126.

[44] WANG W, LI C, ZHANG J, et al. Effects of atomic oxygen treatment on structures, morphologies and electrical properties of $\mathrm{ZnO}$ : Al films. Appl. Surf. Sci., 2010, 256(14): 4527-4532.

[45] CHAVERZ R, IONESCU E, BALAN C, et al. Effect of ambient atmosphere on crosslinking of polysilazanes. J. Appl. Polym. Sci., 2011, 119(2): 794-802.

[46] LI S, ZHANG Y. Effect of synthesis temperature on structure and ceramization process of polyaluminasilazanes. Chinese Journal of Inorganic Chemistry, 2011, 27(5): 943-950.

[47] CHANG Y C, LIU T Z, ZHANG H, et al. Protection of Kapton from Atomic-oxygen Erosion Using a Polysilazane Coating. LIU H W, WANG G, ZHANG G W. Material Science, Civil Engineering and Architecture Science, Mechanical Engineering and Manufacturing Technology II. Switzerland: Trans. Tech. Publications, 2014, 651: $65-68$.

[48] DUO S, CHANG Y C, LIU T, et al. Atomic oxygen erosion resistance of polysiloxane/POSS hybrid coatings on Kapton. Phys. Procedia, 2013, 50: 337-342.

[49] KLEIMAN J I. Surface modification technologies for durable space polymers. MRS Bull., 2010, 35(1): 55-65.

[50] ISKANDEROVA Z A, KLEIMAN J I, GUDIMENKO Y, et al. Surface Modification of Polymers and Carbon-based Materials by ion Implantation and Oxidative Conversion: U.S., 5,683,757. 1997-11-4.

[51] GUDIMENKO Y, KLEIMAN J I, COOL G R, et al. Modification of Subsurface Region of Polymers and Carbon-based Materials: U.S., 5,948,484. 1999-9-7.

[52] GUDIMENKO Y, NG R, KLEIMAN J, et al. Photosilim Surface Modification Treatment of Polymer-based Space Materials and External Space Components. KLEIMAN J, ISKANDEROVA Z A. Protection of Materials and Structures from Space Environment. U.S.: Kluwer Academic Publishers, 2004: 419-434.

[53] ISKANDEROVA Z A, KLEIMAN J I, GUDIMENKO Y, et al. Research Aspects of Scaling-up Implantox Technology for Protec- 
tion of Polymers in Space by Ion Implantation. Protection of Space Materials from the Space Environment. Dordrecht: Springer, 2001: $145-163$.

[54] GU H Y. Surface Activation and Silanization of Polyimide. Shanghai: Shanghai Institute of Ceramics PhD Thesis, 2015.

[55] SHU M, LI Z, MAN Y, et al. Surface modification of poly (4, 4'-oxydiphenylene pyromellitimide)(Kapton) by alkali solution and its applications to atomic oxygen protective coating. Corros. Sci., 2016, 112: 418-425.

[56] LIU K, MU H, SHU M, et al. Improved adhesion between $\mathrm{SnO}_{2} / \mathrm{SiO}_{2}$ coating and polyimide film and its applications to atomic oxygen protection. Colloids Surf. A, 2017, 529: 356-362.

[57] WANG D, GAO Z M, LI Z H, et al. Analysis of erosion effect of environmental factors on polyimide films and coatings. Surface Technology, 2018, 47(1): 123-128.

[58] ISKANDEROVA Z A, KLEIMAN J I, GUDIMENKO Y, et al. Influence of content and structure of hydrocarbon polymers on erosion by atomic oxygen. J. Spacer. Rockets, 1995, 32(5): 878-884.

[59] XIE Y, GAO Y, QIN X, et al. Preparation and properties of atomic oxygen protective films deposited on Kapton by solvothermal and Sol-Gel methods. Surf. Coat. Technol., 2012, 206(21): 4384-4388.

[60] BANKS B A, DE GROH K K, AUER B M, et al. LDEF. Monte Carlo Modeling of Atomic Oxygen Attack of Polymers with Protective Coatings on LDEF. N93-28282, Ohio: NASA, 1993: 1137 1150.

[61] WEAVER A B, KULAKHMETOY M, ALEXEENKO A A. Consistent atomic oxygen model for firect dimulation monte carlo below 1000 kelvin. J. Thermophys. Heat Transfer, 2016: 689-694.

[62] LIU Y, LI G. Numerical simulation on atomic oxygen undercutting of Kapton film in low earth orbit. Acta Astronaut., 2010, 67(3/4): 388-395.

[63] HUANG Y, TIAN X, LÜ S, et al. An undercutting model of atomic oxygen for multilayer silica/alumina films fabricated by plasma immersion implantation and deposition on polyimide. Appl. Surf. Sci., 2011, 257(21): 9158-9163.

[64] BANKS B A, DE GROH K K, KNEUBEL C A. NASA STI Program. Comparison of the Results of MISSE 6 Atomic Oxygen Erosion Yields of Layered Kapton H Films with Monte Carlo Compu- tational Predictions, NASA/TM-2014-218411, Ohio: NASA, 2014.

[65] DUO S W, LI M S, ZHANG Y M. Erosion theoretical and predictive models of atomic oxygen for space materials in low earth orbit. Chinese Journal of Materials Reaserch, 2003, 17(2): 113-121.

[66] LEE C H, CHEN L W. Reactive probability of atomic oxygen with material surfaces in low earth orbit. J. Spacecr. Rockets, 2000, 37(2): 252-256.

[67] LIU T, SUN Q, MENG J, et al. Degradation modeling of satellite thermal control coatings in a low earth orbit environment. Sol. Energy, 2016, 139: 467-474.

[68] CHEN L, LI Z, LEE C H, et al. Unified model for low-earthorbital atomic-oxygen and atomic-oxygen/ultraviolet induced erosion of polymeric materials. Aerosp. Sci. Technol., 2016, 53: 194 206.

[69] VAN DUIN A C T, DASGUPTA S, LORANT F, et al. ReaxFF: a reactive force field for hydrocarbons. J. Phys. Chem. A, 2001, 105(41): 9396-9409.

[70] RAHNAMOUN A, VAN DUIN A C T. Reactive molecular dynamics simulation on the disintegration of Kapton, POSS polyimide, amorphous silica, and teflon during atomic oxygen impact using the reaxff reactive force-field method. J. Phys. Chem. A, 2014, 118(15): 2780-2787.

[71] RAHMANI F, NOURANIAN S, LI X, et al. Reactive molecular simulation of the damage mitigation efficacy of POSS-, graphene-, and carbon nanotube-loaded polyimide coatings exposed to atomic oxygen bombardment. ACS Appl. Mater. Interfaces, 2017, 9(14): $12802-12811$

[72] ZENG F, PENG C, LIU Y, et al. Reactive molecular dynamics simulations on the disintegration of PVDF, FP-POSS, and their composite during atomic oxygen impact. J. Phys. Chem. A, 2015, 119(30): 8359-8368.

[73] GINDULYTE A, MASSA L, BANKS B A, et al. Degradation of Polymers by O (3 P) in Low Earth Orbit. Protection of Materials and Structures from Space Environment. Dordrecht: Springer, 2004: 299-306.

[74] ZHANG H, REN S, PU J, et al. Barrier mechanism of multilayers graphene coated copper against atomic oxygen irradiation. Appl. Surf. Sci., 2018, 444: 28-35. 